\title{
The Spatial Distribution of the Temperature in the Bathtub HaoranSun
}

\author{
North China Electric Power University, Baoding 071000, China.
}

e-mail:2858773155@qq.com

Keywords: Bathtub, Temperature Distribution, Spatial Partitioning Method

\begin{abstract}
We utilized the finite-difference method to establish the discrete distribution model of the two-dimensional bathtub plane at the heating moment. We achieved the simulation of temperature distribution on the two-dimensional plane through numerical analysis, Gauss-Seidel iterative method. By comparing the temperature spatial distribution of heating water on the edge and in the middle of the bathtub, we finally drew the conclusion of the influence of bathtub faucet position on the spatial distribution of water temperature in the bathtub. We optimized the position of the faucet so as to save water. Finally, the water tap is placed in the middle of the water saving effect is better.
\end{abstract}

\section{Introduction}

The way of heating the water in the ordinary bathtub is Injecting hot water into the tub through the hole of the water injection. When the hot water inject into the bathtub, the distribution of the space of the temperature affects the comfort of people bathing. Meanwhile, when the placement of hot water injection holes is different, the distribution of hot water temperature with space also changes. Therefore, for the choice of hot water injection hole, the study of hot water temperature distribution with space in the tub is significant.

\section{Assumptions}

We assume that heat transfer in the bathtub corresponds to the heat transfer between the bath water and tap water. Explanation: Compared to the influence of heat transfer between the bath water and tap water, other heat transfer phenomena can be ignored.

We view temperature distribution of bathtub as a 2D steady state temperature field. Explanation: The model is established to simplify the temperature distribution; this choice of thought simplifies the analysis of the particular problem.

We regard the bathtub as a rectangle. Explanation: we simplify the shape of the object to make a more clear understanding.

\section{The Spatial Partitioning Method}

We research Two-dimensional plane of bathtub. Consider the Planar Regions of bath in the initial moment, (The moment the tap just put out the water.) revised exponential model, get temperature distribution model of bathtub. Firstly, we apply spatial partitioning method to Planar Regions of Bath. This research sets two-dimensional water pool plane as research object, and the temperature distribution in the pool of the initial time (i.e. the moment when water pipe releases water) is considered. First of all, the two-dimensional pool plane is divided into discrete partitions. The $X$ axis is set up along the width, and the $Y$ axis is established along the length. The width is divided into 20 equal parts, forming $M=21$ nodes, the distance between two adjacent nodes is $\Delta X$. The length is divided into 30 equal parts, forming a $N=31$ nodes, the distance between two adjacent nodes is $\Delta y$. In this way, a mesh area of $20 * 30$ is formed on the plane. As shown in figure 8 . The temperature of each node can be expressed as $t_{\mathrm{m}, \mathrm{n}}$. [1] 


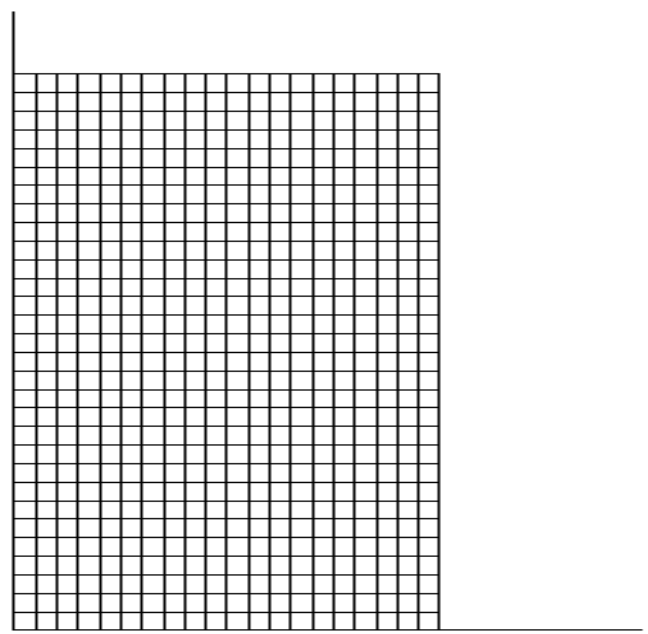

Figure 1 . A mesh area of $20 * 30$ is formed on the plane.

\section{Build Discrete Node Equation}

Using the method of heat balance, discrete node equation was established. We regard the bathtub as a rectangle. The assumption is that the temperature of the left, right and back surfaces is $35^{\circ} \mathrm{C}$. The faucet is located on the front surface of the rectangle (water). Therefore the temperature of the front surface is $40^{\circ} \mathrm{C}\left(T \mathrm{~s}=40^{\circ} \mathrm{C}\right)$. Using the method of heat balance, we have a relation among parameters.[2]

Connection between internal nodes

$$
\lambda \Delta y \frac{t_{m-1, n}-t_{m, n}}{\Delta x}+\lambda \Delta y \frac{t_{m+1, n}-t_{m, n}}{\Delta x}+\lambda \Delta x \frac{t_{m, n+1}-t_{m, n}}{\Delta y}+\lambda \Delta x \frac{t_{m, n-1}-t_{m, n}}{\Delta y}=0
$$

Connection between internal nodes of front surface

$$
\lambda \frac{\Delta y}{2} \frac{t_{m-1,1}-t_{m, 1}}{\Delta x}+\lambda \frac{\Delta y}{2} \frac{t_{m+1,1}-t_{m, 1}}{\Delta x}+h \Delta x\left(t_{s}-t_{m, 1}\right)+\lambda \Delta x \frac{t_{m, 2}-t_{m, 1}}{\Delta y}=0
$$

\section{Solving Discrete Equation}

Solution of this discrete equation is iterative method; the specific is based on Seidel - Gauss iterative method for solving

The discrete equation is organized into the form of the explicit function of the temperature in each node. As you can see below:

$$
\begin{gathered}
t_{m, 1}=\left[\frac{\Delta y}{2 \Delta x}\left(t_{m-1,1}+t_{m+1,1}\right)+\frac{\Delta x}{\Delta y} t_{m, 2}+\frac{h \Delta y}{\lambda} t_{s}\right] /\left(\frac{\Delta y}{\Delta x}+\frac{\Delta x}{\Delta y}+\frac{h \Delta y}{\lambda}\right) \\
m=2,3, \ldots \ldots, M-1
\end{gathered}
$$

For unknown node temperature, the first solution is $38^{\circ} \mathrm{C}$. The heat transfer coefficient of water can be found by looking up table $(\lambda=0.599 \mathrm{~W} / \mathrm{m} \cdot \mathrm{K})$. Heat transfer between water and the front surface corresponds to the heat transfer between the bath water and tap water. So the heat transfer coefficient $h=0.599 \mathrm{~W} /(\mathrm{m} \cdot \mathrm{K})$. Thus, we can get temperature distribution image. As you can see as below:[3] 


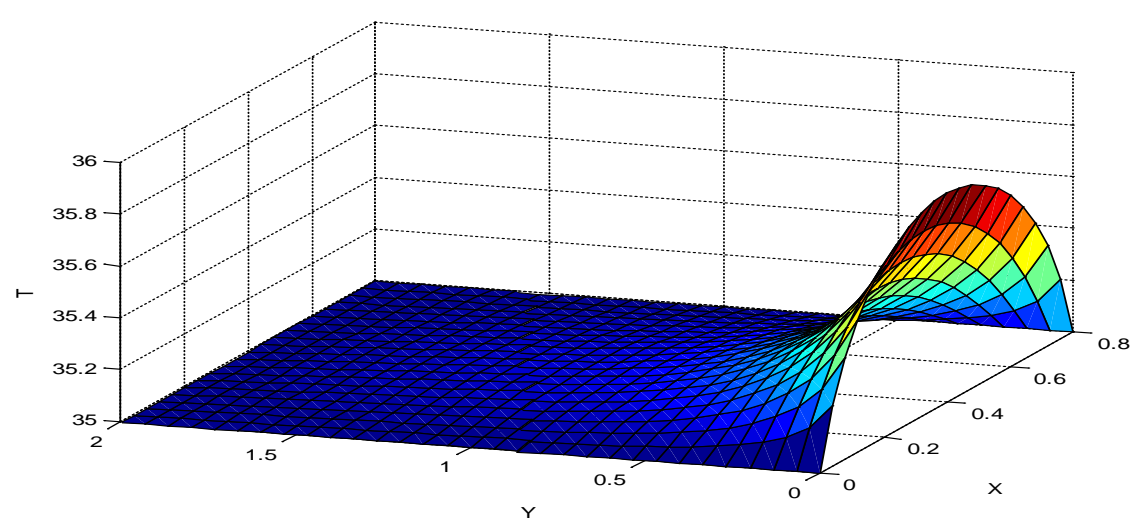

Figure 2. The image of initial bath temperature distribution.

\section{Further Analysis of the Results}

Temperature distribution of bathtub was observed by changing the initial value of the outlet temperature of the water tap (Ts):[4]. From Figure 3, we can conclude that the temperature of the water is improved in the initial time, with the increasing Ts. The closer to the front surface, temperature gradually becomes increasing trend.

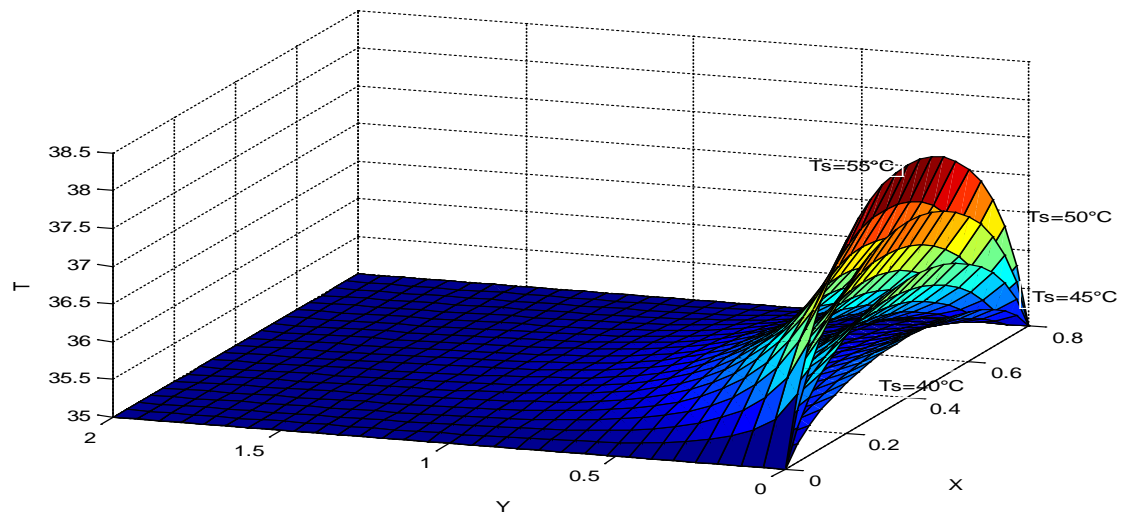

Figure 3. comparison chart

So, we can improve temperature of bathtub by increasing the outlet temperature of the water tap. With time going by, temperature distribution will gradually tend to be uniform. Heating effect will be enhanced, heating time will be shortened and water will be saved by doing so.

To explore Effect of tap location on temperature distribution, the outlet temperature of the tap(Ts) is $55^{\circ} \mathrm{C}$. (At this point, the effect is modified best). The tap is placed in the middle of the bathtub. Temperature distribution of bathtub was observed by changing the location of the tap.[5]

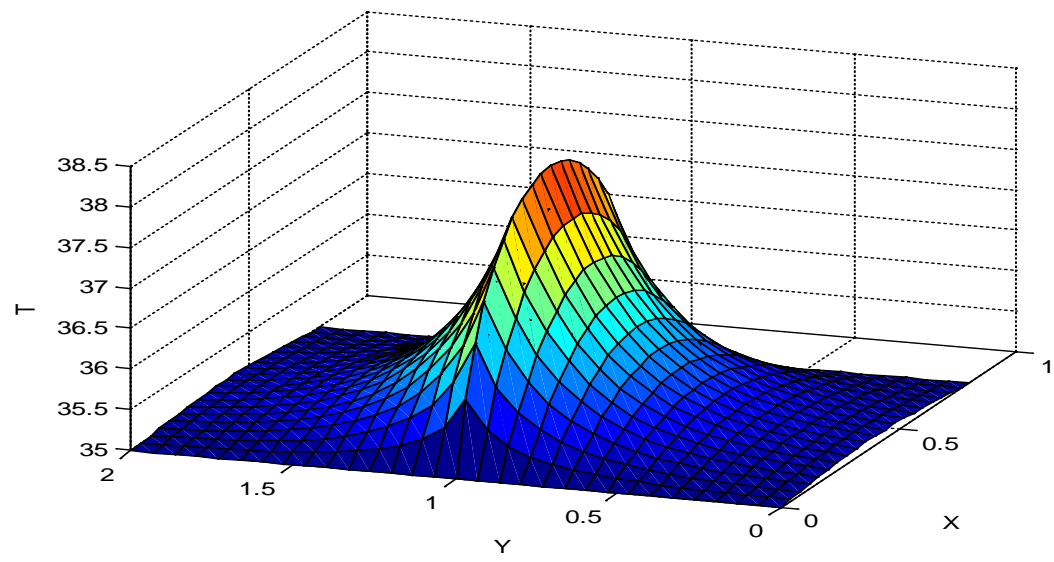

Figure 4. Place the tap in the middle 
We can see from Figure4, high-temperature water area becomes bigger. That is to say the efficiency of healing becomes better. Therefore, the heating effect can be increased by placing the tap in the middle position. Heating time will be shortened and water will be saved by doing so.

\section{Conclusions}

We can get the spatial distribution of the temperature in the bathtub by using the model. Apparently, we can find that Different positions of water injection holes can lead to changes in temperature distribution. As a result, the comfort of the bath will also change. It can be obtained by verification that our model is correct and generic.

\section{References}

[1] J. Xiao and G. Xiao, "Equivalence principle for 3D anisotropic multi-physics model building," 2012 Asia Pacific Microwave Conference Proceedings, Kaohsiung, 2012, pp. 1301-1303.doi: 10.1109/APMC.2012.6421902

[2]Yan Feng Liu, Zhang Yang Gao, Xiu Jiuliang, heat transfer [M] CHINA ELELCTRIC POWER PRESS 2.2015.

[3]Huaizhu Wang, High Efficient and Stable Numerical Method for Three-dimensional Unsteady Heat Conduction Problems, Journal of Hebei North University(Natural Science Edition), October 2009, Pages1-4.

[4] Kai Xu, Lina Shi, Dongyin Wu, "Numerical Simulation of Thermal Problem Based on MATLAB,” Journal of Shanghai Engineering University, 2016,04:353-358.

[5] Changshen Ye, "Application of MATLAB in Steady State and Dynamic Thermal Process Analysis,” Computer and Applied Chemistry, 2006,10:986-990. 